\title{
GROWTH-RELATED CONSTRAINTS ON DIET SELECTION BY SUNFISH ${ }^{1}$
}

\author{
WILLIAM E. WALTON \\ Center for Great Lakes Studies, University of Wisconsin-Milwaukee, 600 East Greenfield Avenue, \\ Milwaukee, Wisconsin 53204 USA
}

Nelson G. Hairston, JR.

Section of Ecology and Systematics, Cornell University, Ithaca, New York 14853 USA

\author{
JAMES K. WeTtereR ${ }^{2}$ \\ Department of Biology, Princeton University, Princeton, New Jersey 08544 USA
}

\begin{abstract}
Diet selectivity by the planktivorous bluegill sunfish (Lepomis macrochirus) is correlated with the fishes' visual resolution. Poor visual capabilities are thought to impose a constraint on the ability of small sunfish to choose prey. Li et al. (1985) suggested that sunfish subjected to conditions of poor visual resolution select prey in accordance with a model in which prey choice is determined by apparent prey size. Further, enhanced visual resolution permitted large sunfish to assess absolute prey size more accurately than could smaller conspecifics and, consequently, large-sized sunfish consumed relatively more large prey than was predicted by the apparent size model. Here, we examine the visual capabilities and prey selection of very small sunfish, extend the analyses of $\mathrm{Li}$ et al. by determining the optimal diet sets for the fish used in their study, and compare prey selection by all sunfish size categories to that predicted by the apparent size and optimal foraging models. Contrary to the conclusions of $\mathrm{Li}$ et al., small sunfish $(<20 \mathrm{~mm}$ standard length [SL]) were more selective than was predicted by the apparent size model and preferentially attacked small-sized Daphnia. The optimal foraging "prey" model predicted correctly the sizerelated changes in mean size of prey attacked by sunfish, whereas the apparent size model predicted a preference for larger Daphnia for all sizes of fish. Yet, the observed diet breadths were always larger than those predicted by the optimal foraging model. The visual system and energy considerations constrain prey selection in small sunfish but operate in opposite fashions.
\end{abstract}

Key words: apparent size; Daphnia; Lepomis; optimal foraging; predation; prey selection; visual resolution.

\section{INTRODUCTION}

The abilities of fish to detect and capture prey change as fish grow. Neuroanatomical studies have shown that visual resolution increases with fish size as both the eye and the physical relationships among its components change during postembryonic development (Blaxter and Staines 1970, Johns and Easter 1977, Hairston et al. 1982, Neave 1984, Fernald 1988). Similar increases in visual resolution have been found in behavioral studies (Hairston et al. 1982, Schmidt and O'Brien 1982, Breck and Gitter 1983, Li et al. 1985). In the bluegill sunfish, Lepomis macrochirus Rafinesque, visual resolution increases nonlinearly with fish size. Visual resolution changes markedly in small-sized fish (27-50 mm: standard length [SL]) and the change diminishes in larger fish (>50 mm SL) (Breck and Gitter 1983, Li et al. 1985). In addition to size-related

\footnotetext{
' Manuscript received 19 November 1990; revised 18 April 1991; accepted 30 April 1991; final version received 28 May 1991.

${ }^{2}$ Present address: Museum of Comparative Zoology, Harvard University, Cambridge, Massachusetts 02138 USA.
}

changes in visual capabilities, traits that influence a fish's ability to capture prey, such as swimming speed (Mittelbach 1981, Blaxter 1986) and mouth gape (Werner 1974, Hunter 1981, Lemly and Dimmick 1982, Schmitt and Holbrook 1984), and ability to digest food (i.e., Rutilus rutilus L.; Hofer and Nasir Uddin 1985, Mark et al. 1989) also increase directly with fish size (Miller et al. 1988).

Models of diet selection, whether based on simple encounter principles or on optimality theory, require knowledge of a forger's ability to detect and capture prey. The predictions of encounter-based and optimal foraging models differ, however, as a function of fish size. O'Brien et al. (1976) proposed that planktivorous sunfish select among simultaneously encountered prey by attacking the apparently largest prey. In its simplest form, this Apparent Size Model (ASM) predicts that the diets of sunfish reflect the predator's encounter probabilities with prey whose sizes and abundances differ in the visual field (O'Brien et al. 1976, Wetterer and Bishop 1985). By selecting the apparently largest prey, sunfish would exhibit adaptive shifts in foraging behavior by including an increasing proportion of large 
prey in the diet as prey density increases (O'Brien et al. 1976, Eggers 1982).

Subsequent studies revealed that the predictions of the ASM differed from those suggested by O'Brien et al. (1976). Butler and Bence (1984) concluded that prey selection by apparent size should lead to constant prey preferences independent of prey density and suggested that prey depletion and assumptions about the way bluegills detect and select prey accounted for an apparent increase in preference for large Daphnia at high prey densities relative to low prey densities (Werner and Hall 1974, O'Brien et al. 1976). Wetterer and Bishop (1985) found that the predictions of the ASM are dependent on the size and shape of the reactive fields, and on relative and absolute prey densities, particularly at low prey densities. Whereas prey density and visibility will influence model predictions, the ASM clearly predicts no differences in prey selection with changes in fish body size (Werner et al. 1983).

Optimal foraging models differ from the ASM in that prey selection is governed by energy maximization considerations that change with fish size and foraging abilities (Werner et al. 1983). In optimal foraging models (Mittelbach 1981, Werner et al. 1983), size-related differences in prey detection are incorporated as search time and its cost. In addition to the binary outcome of the predation event (the prey is either attacked or ignored), optimal foraging theory adds the costs incurred by a forager during prey capture. Even when capture and ingestion are successful, a predator incurs energy costs during the time spent handling prey. The fact that the predictions of encounter and optimal foraging models differ as function of fish size provides a means to discriminate between the models.

$\mathrm{Li}$ et al. (1985) used this approach to compare the apparent size model's predictions and the observed diets of bluegill sunfish between 38 and $76 \mathrm{~mm} \mathrm{SL}$ (mean standard length). They found that large- $(76 \mathrm{~mm}$ SL) and medium-sized (60 mm SL) sunfish specialized on large prey to a greater degree than was predicted by the apparent size model. The diets of small-sized ( 38 $\mathrm{mm} \mathrm{SL}$ ) fish differed little from those predicted by the apparent size model. Li et al. (1985) suggested that bluegill sunfish subjected to poor visual conditions feed according to apparent size. Poor visual conditions could result from a large average distance to prey, low prey density, or limitations imposed by retinal anatomy. Situations resulting in good visual conditions, such as a short average distance to prey, high prey densities, or enhanced visual resolution, were interpreted by $\mathrm{Li}$ et al. (1985) to permit more complex feeding behavior.

Because visual resolution is directly related to sunfish size (Hairston et al. 1982, Breck and Gitter 1983, $\mathrm{Li}$ et al. 1985), Li et al.'s conclusion implicitly suggests that, as fish grow, sunfish switch foraging decision rules. Small sunfish have poor visual capabilities and should feed according to the apparent size model. Larger sunfish have comparatively better visual capabilities and are predicted to make foraging decisions according to rules that are more complex than the ASM (e.g., optimal foraging behavior). However, Li et al. (1985) did not explicitly test the optimal foraging model of Mittelbach (1981).

Here, we determine the visual capabilities of fingerling sunfish (8-33 mm SL) and compare prey selection by these very small fish to that predicted using the apparent size model of Wetterer and Bishop (1985) and the optimal foraging model of Mittelbach (1981). If the predictions of $\mathrm{Li}$ et al. (1985) are correct, and fingerling sunfish have poor visual capabilities, then very small sunfish should feed according to the apparent size model.

Second, we develop explicit optimal foraging theory (OFT) predictions for comparison with the empirical results for the larger sunfish used by $\mathrm{Li}$ et al. (1985). We use these OFT predictions, the ASM predictions for the larger sunfish (Li et al. 1985), the predictions of both models for the fingerling sunfish and the observed prey selection of 10 to $>76 \mathrm{~mm} \mathrm{SL}$ sunfish to determine whether growing sunfish switch foraging decision rules. We ask, do the foraging decision rules of sunfish change from the ASM to OFT as fish size increases or does either model more accurately predict the size-related shifts of prey selection by sunfish?

In contrast to the predictions of prey selection by the two models for comparatively large-sized sunfish, the predictions of prey selection based on encounter rates and based on foraging profitability diverge for very small sunfish. The predictions of prey selection based on encounter rates (ASM) and those based on foraging profitability (OFT) are usually similar for large bluegills because the fish are very large relative to the zooplankton prey, and all prey size categories are captured easily. The largest prey are the most visible and are also the most profitable to sunfish foraging in the experimental conditions found in many previous studies. As fish size decreases, the predictions of prey selection based on encounter rates may differ increasingly from, and may counter, the predictions of prey selection based on foraging profitability. Larger zooplankton are always more visible, yet, for very small sunfish, larger prey have long handling times, and hence may not be more profitable than smaller prey. Thus, by taking advantage of the substantial shifts in the foraging capacity as fish grow, we are better able to distinguish empirically between the two models.

\section{Materials AND Methods}

\section{Study organisms}

Juvenile sunfish (Lepomis spp.: 8-33 mm SL) were dipnetted from Dryden Lake, Tompkins County, New York, and maintained in 20-L plastic buckets on a daily ration of mixed zooplankton. Although it is not possible to distinguish visually between closely related species of Lepomis in the smallest sizes, electrophoretic 
analyses using loci known to be diagnostic revealed that the majority $(80 \%)$ of the fish used here were bluegill sunfish, $L$. macrochirus, and the remainder were pumpkinseeds, L. gibbosus (Linnaeus) (R. Dawley, personal communication; see Dawley 1987 for methods).

Mittelbach (1984, 1986) and Osenberg et al. (1988) showed that small bluegill and pumpkinseed sunfish are ecologically analogous. Small individuals of these species competed exploitatively within the littoral zone (Mittelbach 1986) and exhibited similar growth rate responses to within-lake environmental variation (20$55 \mathrm{~mm}$ bluegill and $20-40 \mathrm{~mm}$ pumpkinseed: Osenberg et al. 1988). We did not observe two distinct groupings in any of the behavioral measures discussed below and, therefore, assume that any difference in the visual capabilities and prey selection of the small sunfish did not have an important influence on our results.

\section{Reaction distance}

Reaction distances were measured for 36 fish in a 30 by $40.5 \mathrm{~cm}$ plexiglass box positioned over a grid of 1-cm squares. Water depth was either $2 \mathrm{~cm}$ (for 8-19 $\mathrm{mm} \mathrm{SL} \mathrm{fish)} \mathrm{or} 4 \mathrm{~cm}$ (for 25-33 mm SL fish), and ambient light levels at the water surface were 4-4.5 $\mu \mathrm{mol} \cdot \mathrm{s}^{-1} \cdot \mathrm{m}^{-2}$ provided by two, $40-\mathrm{W}$, cool-white fluorescent lamps.

Overhead video recordings were used to determine the $x$ and $y$ coordinates of (1) the fish's head and (2) the prey at the time the fish began pursuit of the prey. Fish initiated pursuit by orienting the long axis of the body towards the prey (Vinyard and O'Brien 1976). Reaction distance, $d$, was defined as the distance between points (1) and (2). The video camera was suspended $260 \mathrm{~cm}$ above the water surface; hence, errors in the measured reaction distance due to parallax were $<3 \%$ (Hairston et al. 1982).

\section{Prey selection}

Diet choice experiments were run using equal and high densities $(20 / \mathrm{L})$ of three $D$. pulex size classes. Thirty-five sunfish (10-19 mm SL) were offered $D$. pulex of the following size categories: small, $0.82 \pm$ $0.08 \mathrm{~mm}$; medium, $1.58 \pm 0.13 \mathrm{~mm}$; and large, 2.16 $\pm 0.09 \mathrm{~mm}$ (mean total length $\pm 1 \mathrm{sD}$ ). Eighteen sunfish (25-33 mm SL) were offered three $D$. pulex size categories: small, $1.27 \pm 0.08 \mathrm{~mm}$; medium, $1.82 \pm 0.13$ $\mathrm{mm}$; and large, $2.53 \pm 0.12 \mathrm{~mm}$. Prey were sorted using a series of sieves and then sorted additionally under a dissecting microscope.

The experiments were run in a circular, white plastic arena $27 \mathrm{~cm}$ in diameter and filled with $5.25 \mathrm{~cm}$ of net-filtered (79- $\mu \mathrm{m}$ mesh opening) Dryden Lake water (total volume $3 \mathrm{~L}$ ). Water temperatures ranged between $21^{\circ}$ and $22^{\circ} \mathrm{C}$. Fluorescent lighting provided a photon flux density of $7-8 \mu \mathrm{mol} \cdot \mathrm{s}^{-1} \cdot \mathrm{m}^{-2}$ at the water surface. Individual fish were allowed to consume five prey items or were observed for $30 \mathrm{~min}$, whichever came first. A sequence of strikes at the same prey was considered a single attack, and successful and unsuccessful captures were recorded. The size distributions of the uneaten prey were determined using a dissecting microscope.

The diets predicted by the apparent size model were calculated using the computer simulation of Wetterer and Bishop (1985). Reaction distances to prey of different sizes were calculated from video recordings. Other parameters used in the simulation, such as the abundance and size distributions of prey and the physical characteristics of the experimental environment, are given above. The observed attacks and those predicted by the apparent size model were compared by chisquared tests.

The optimal foraging model of Mittelbach (1981) was used to derive the optimal diets predicted for different-sized sunfish. The net rate of energy intake $\left(E_{n} /\right.$ $T$, in joules per second) was calculated as:

$$
E_{n} / T=\frac{\sum_{i=1}^{n}\left(B_{i} E_{i} S_{i}\right)-C_{s}}{1+\sum_{i=1}^{n} B_{i} H_{i} S_{i}},
$$

where energy intake is

$$
E_{i}=A e_{i}-C_{h} H_{i}
$$

and where $B_{i}$ is the encounter rate for prey size class $i$ (number per second); $H_{i}$ is the handling time of prey size class $i$ (in seconds); $C_{h}$ is the energy cost of handling prey (in joules per second); $C_{s}$ is the energy cost of searching (in joules per second); $A$ is the assimilable fraction of energy content (taken to be 0.7 as per Mittelbach 1981); and $e_{i}$ is the energy content of prey size class (in joules). Swimming speeds during searching and handling were taken to be 1.1 and $0.5 \mathrm{~m} / \mathrm{min}$, respectively (from Mittelbach 1981). Regression equations describing the relationships between predator size and costs, prey size and energy content, and encounter rate were taken from Mittelbach (1981).

Small sunfish do not always successfully capture Daphnia and, because encounter rates were extrapolated to smaller sized sunfish than were used by Mittelbach, encounter rates may not be described adequately by Mittelbach's regression. Hence, size-related probabilities of prey capture $\left(S_{i}\right)$ were added to the optimal foraging model. For each fish size category, $S_{i}$ was calculated from the prey selection experiments as captures/attacks. In order to address the potential problem of linear extrapolation of encounter rates to small sunfish, we adjusted $B_{i}$ by decreasing the encounter rates for $29 \mathrm{~mm}$ SL sunfish in proportion to the truncated, visual volumes of sunfish $<29 \mathrm{~mm} \mathrm{SL}$. We assumed that encounter rates for sunfish $\geq 29 \mathrm{~mm}$ SL were described accurately by Mittelbach's regression. 
Werner (1974) and Mittelbach (1981) showed that the handling time per item was a function of relative prey size ( $l / L$ : prey length/fish length). Since our fish were smaller than the smallest individuals studied by Werner (24 mm SL) and Mittelbach (33 mm SL), we examined the relationship between handling time per prey item and relative prey size for 31 sunfish between 13 and $18 \mathrm{~mm}$ SL. The fish were fed three Daphnia pulex size classes $(0.73 \pm 0.04,1.86 \pm 0.10$, and 2.12 $\pm 0.07 \mathrm{~mm}$ : mean length $\pm 1 \mathrm{sD}$ ) at densities ranging between 110 and 225 prey/L. Each prey size class was offered separately. Fish were allowed to feed for short periods $(<180 \mathrm{~s})$. The time required to attack all prey items was recorded with a stopwatch. Handling time per prey item $(H)$ was calculated as: (total number of prey consumed minus one)/(time of the feeding bout).

Since the fish often required several minutes to acclimate to the experimental surroundings, the time of the foraging bout was the period during which fish consumed prey in rapid succession. By providing the fish with prey at high densities and recording handling time as above, we intended to minimize search time (essentially zero) and determine the average handling time for each Daphnia size class by fish of known size. We did not quantify handling time per prey item by measurements using individual prey because we could not discern when a prey was swallowed.

The optimal diet for a given size of predator is that subset of available prey sizes that maximizes the net rate of energy intake (Mittelbach 1981). Prey size classes were ranked from highest to lowest $E_{i} / H_{i}$, and added to the diet until the ratio $E_{n} / T$ was maximized. Handling times derived from our experiments above were used to calculate prey profitability for fish $\leq 19 \mathrm{~mm}$ SL. For sunfish $>19 \mathrm{~mm}$ SL, prey profitability was calculated using the relationship between handling times and relative prey length given by Mittelbach (1981):

$$
H=0.536 e^{18.488 \text { (prey length/fish length). }}
$$

In our experiments, relative prey size exceeded the critical ratio $(0.034)$ below which handling time remains constant (Mittelbach 1981).

The optimal diets of the fingerling sunfish were determined at 1-mm (SL) intervals. The expected diet for each predator size category was calculated as the composite of the optimal diets for the sunfish in each predator size grouping. By weighting the expected diet in this manner, partial preferences could occur within some predator groups. The expected diets for sunfish $>35 \mathrm{~mm}$ SL were calculated using the average length of the three predator groups given by Li et al. (1985). Since reaction distances of the fish exceeded one-half the depth of the experimental environment, the distribution of expected attacks was corrected for reductions in visual field volume using $2 \pi a\left(d^{2}-a^{2} / 3\right)$ (Werner and Hall 1974, Wetterer and Bishop 1985), where $d$ is the reaction distance and $a$ is equal to one-half the depth of the experimental environment.
TABLE 1. The reaction distances (RD) and probabilities of successful prey capture $\left(S_{i}\right)$ for the four smallest sunfish size classes (standard lengths, SL).

\begin{tabular}{|c|c|c|c|c|c|c|}
\hline \multirow{3}{*}{$\begin{array}{c}\text { Fish } \\
\text { size } \\
(\mathrm{mm} \\
\mathrm{SL})\end{array}$} & \multicolumn{6}{|c|}{ Prey size category } \\
\hline & \multicolumn{2}{|c|}{ Small } & \multicolumn{2}{|c|}{ Medium } & \multicolumn{2}{|c|}{ Large } \\
\hline & $\begin{array}{l}\mathrm{RD} \\
(\mathrm{cm})\end{array}$ & $S_{i}$ & $\begin{array}{l}\mathrm{RD} \\
(\mathrm{cm})\end{array}$ & $S_{i}$ & $\begin{array}{l}\mathrm{RD} \\
(\mathrm{cm})\end{array}$ & $S_{i}$ \\
\hline 11.2 & 2.9 & 0.60 & 5.6 & 0.10 & 7.7 & 0 \\
\hline 14.1 & 3.7 & 0.72 & 7.1 & 0.32 & 9.7 & 0.13 \\
\hline 16.6 & 4.3 & 0.79 & 8.4 & 0.65 & 11.4 & 0.28 \\
\hline 29.3 & 13.6 & 1.00 & 19.5 & 1.00 & 27.1 & 1.00 \\
\hline
\end{tabular}

\section{RESULTS \\ Reaction distance}

Reaction distances for the four small sunfish size categories increased directly with fish length and prey size (Table 1). The reaction distances for $29.3 \mathrm{~mm}$ sunfish were 3-4 times as large as those for $11.2 \mathrm{~mm}$ sunfish. Within a particular sunfish size category, reaction distances for the largest Daphnia were $\geq 2$ times as large as for the smallest Daphnia.

\section{Prey selection}

In order to compare the observed attacks with those predicted by the apparent size model, fish were separated into four size categories (mean size: 11.2, 14.1, 16.6 , and $29.3 \mathrm{~mm} \mathrm{SL}$ ) based on the propensity and success of sunfish attacks on the largest Daphnia size class. The largest Daphnia were attacked and captured rarely by the smallest sunfish (11.2 mm: range 10-12 $\mathrm{mm} \mathrm{SL} ; n=11$ ), whereas, large Daphnia were attacked and captured frequently by the largest sunfish $(29.3$ $\mathrm{mm}$ : range 25-33 mm SL; $n=18$ ). Daphnia's predator evasion behavior (a spiral swimming pattern; $\mathrm{Li}$ and Li 1979) was very effective against small sunfish. During multiple attacks at a single prey, small sunfish often were unable to strike successfully. In the $14.1 \mathrm{~mm}$ (range 13-15 mm SL, $n=10$ ) and $16.6 \mathrm{~mm}$ (range $16-19 \mathrm{~mm}$ SL, $n=12$ ) sunfish size categories, the frequencies of attacks and captures increased directly with fish size and were intermediate to those observed in the $\mathbf{1 1 . 2}$ and $29.3 \mathrm{~mm}$ sunfish.

The distribution of attacks by the three smallest fish size categories differed significantly from the predictions of the apparent size model (Fig. 1: $\chi^{2}[11.2 \mathrm{~mm}]$ $=1017.50 ; \chi^{2}[14.1 \mathrm{~mm}]=404.17 ; \chi^{2}[16.6 \mathrm{~mm}]=$ 131.56]; df for each comparison $=2, P<.01$ ). Relative to large and medium Daphnia, small Daphnia were attacked more often than expected. Preference for small Daphnia declined with increasing fish size; the smallest sunfish deviated most from the apparent size model and the two intermediate-sized sunfish groups (14.1 and $16.6 \mathrm{~mm} \mathrm{SL}$ ) were progressively less disparate (Fig. 1). The distribution of attacks by the largest sunfish 

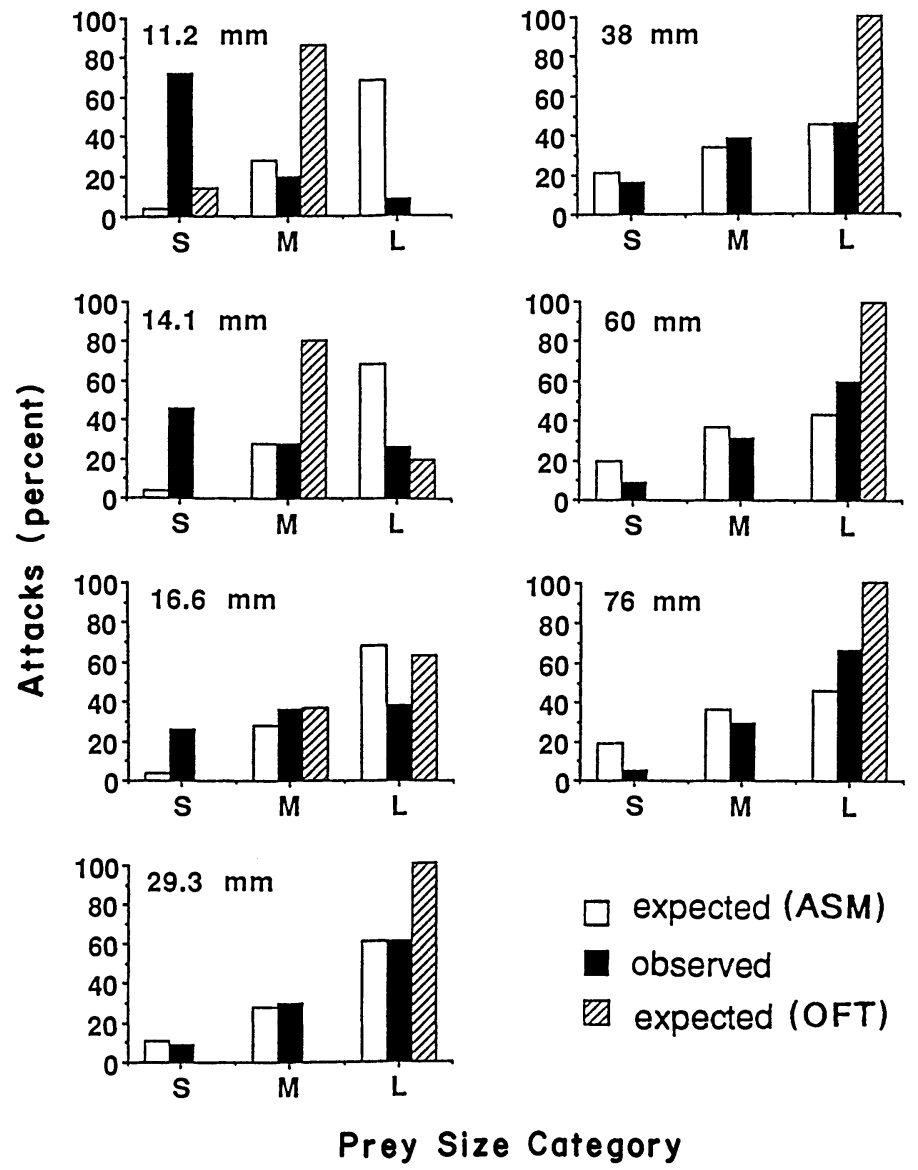

Fig. 1. Distributions of sizes of Daphnia pulex attacked by sunfish (observed) and the distributions of attacks predicted by the apparent size model (ASM) and the optimal foraging theory (OFT) model. The data for 38, 60, and $76 \mathrm{~mm}$ sunfish in the observed and ASM histograms were taken from $\mathrm{Li}$ et al. (1985).

used in our study (29.3 mm SL) did not differ significantly from the apparent size model predictions (Fig. $\left.1: \chi^{2}=0.34, \mathrm{df}=2, P>.05\right)$.

As fish size increased beyond $29 \mathrm{~mm}$ SL, sunfish diets again deviated from the apparent size model predictions. Li et al. (1985) showed previously that the diets of 60 and $76 \mathrm{~mm}$ sunfish included relatively more large prey than was predicted by the apparent size model, but the distribution of prey size categories in the diets of $38 \mathrm{~mm}$ sunfish was similar to that predicted by the apparent size model (Fig. 1).

In the present study, sunfish $<12 \mathrm{~mm}$ SL did not attack large prey when smaller prey were available (selection experiments), even though the largest Daphnia were certainly the most visible. These small sunfish pursued and did occasionally attack large Daphnia when they were the only prey available in the reaction distance experiments. When alternative small prey were present, small sunfish made a postencounter-preattack decision not to attack the largest Daphnia. One potential reason for this choice is that handling times of 2.2 $\mathrm{mm}$ Daphnia are large and, consequently, energy return may be small. If this were true, then the sunfish might forage in accordance with OFT predictions. We examine the predictions of the optimal foraging model below.

Handling times of Daphnia by small sunfish were about twofold longer than those predicted by extending Mittelbach's (1981) regression for handling time vs. relative prey size beyond the minimum fish size he studied. For sunfish $<20 \mathrm{~mm}$ SL, the relationship between handling time $(H)$ and relative prey length $(l / L)$ appears to be a complex function of relative prey size (Fig. 2). Previous studies found that handling time increased exponentially above a critical ratio of prey length/fish length (0.034: Mittelbach 1981, Werner et al. 1983). For sunfish $<20 \mathrm{~mm} \mathrm{SL}$, an exponential function $\left(H=0.566 e^{21.83(/ / L)} ; r^{2}=0.75\right)$ underestimated prey handling times when relative prey size was large $(>0.13)$. The relationship between ln-transformed handling times and relative prey length was described better by a logarithmic equation, 


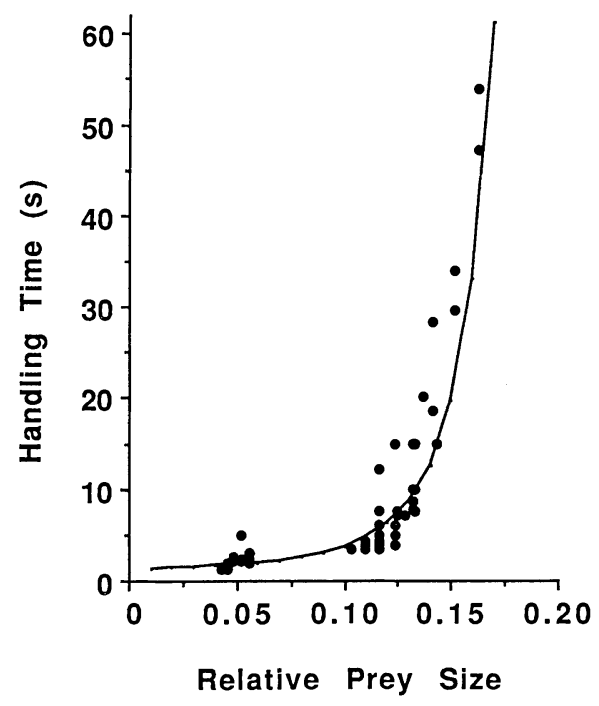

FIG. 2. Prey handling time $(H)$ as a function of relative prey size. Fish sizes ranged between 13 and $18 \mathrm{~mm}$. The curve was fitted by the regression equation: $\ln H=0.264$. $10^{\text {(7.0151 } \text { relative prey length). }}$

$\ln H=0.264 \cdot 10^{(7.0151 \cdot \text { Relative prey length) }}$

$\left(r^{2}=0.90\right)$, than by the exponential equation given above. We used this relationship in the optimal diet model for sunfish $<20 \mathrm{~mm}$ SL (Eq. 1).

The probabilities of successful prey capture were $<1.0$ for sunfish $<20 \mathrm{~mm} \mathrm{SL}$ (Table 1). Capture success increased directly with fish size and inversely with prey size. The probabilities of successful prey capture were 1.0 for sunfish $>20 \mathrm{~mm}$ SL.

Sunfish in all size categories attacked small Daphnia more often than expected and included prey outside the optimal diet set in their diets (Fig. 1). However, the optimal foraging model predicted correctly the change in selectivity from small to large prey as fish size increased. The correlation between the predicted and observed mean prey size attacked was better for the optimal foraging model $(r=0.97, P<.001)$ than for the ASM ( $r=0.56, P>.19$; Fig. 3). Unlike the regression for OFT, where the slope is nearly one, the regression for the ASM is comparatively flat and has a negative slope.

Although the proportion of attacks directed at large and medium Daphnia increased directly with fish size, the optimal foraging model failed to predict qualitatively the breadth of fish diets. The diet breadths of all sunfish size categories predicted by the optimal foraging model were narrower than those observed (Fig. 1).

The predicted diets of the optimal foraging model changed insignificantly when encounter rates were decreased in proportion to declining visual volumes of the three smallest sunfish size classes. Only the diet breadths of the 16.6-mm sunfish increased slightly from those predicted by the original model (expected pro- portions of small : medium : large prey in the diet $=$ $0.1: 0.42: 0.48)$ and the expected mean size of prey attacked increased to $1.78 \mathrm{~mm}$ from $1.70 \mathrm{~mm}$. The optimal foraging model still explained $93 \%$ of the variation in sunfish attacks $\left(y=0.477+0.889 x, r^{2}=0.93\right.$; cf. Fig. 3).

Because Li et al.'s (1985) data were based on gut contents and, because prey sometimes eluded the smallest sunfish, the mean prey size attacked differed from the mean prey size consumed, we repeated the comparison between the expected and observed diets using the prey captured by the four smallest sunfish size categories. The correlation between the predicted and observed mean prey size consumed was again better for the optimal foraging model $(r=0.93, P<.001)$ than for the ASM $(r=0.67, P>.1)$.

\section{DisCUSSION}

Previous studies suggest that the ASM accurately predicts the distributions of prey in the diets of sunfish feeding at low prey densities (Butler and Bence 1984, Wetterer and Bishop 1985). However, the ASM (O'Brien et al. 1976) predicts that the mean prey size

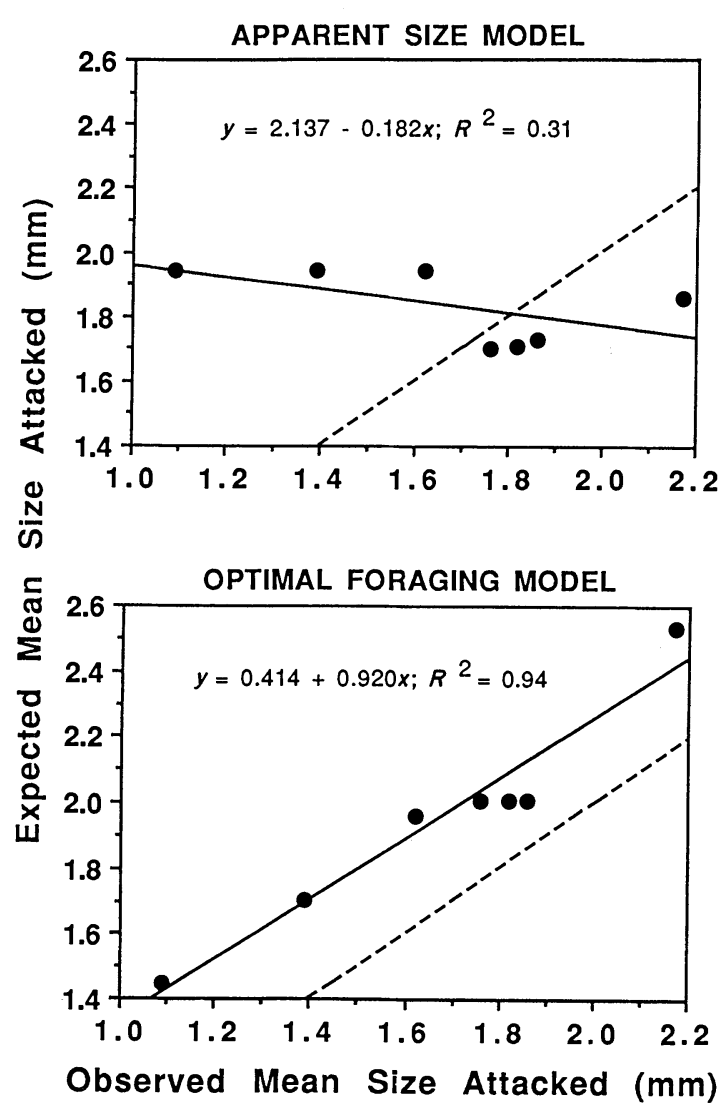

FIG. 3. The relationships between the predicted and the observed mean prey size attacked by 10 to $76 \mathrm{~mm} \mathrm{SL} \mathrm{sunfish}$ for the ASM and OFT models. The solid line is the best fitting least squares regression. The broken line indicates the expected relationship for an exact correspondence between predicted and observed mean prey size attacked. 
attacked (or consumed) and the distribution of sunfish attacks do not change with fish size for sunfish feeding on similar prey assemblages (Werner et al. 1983, Butler and Bence 1984). At high prey densities, large-sized prey are overrepresented in bluegill diets and this selectivity shift agrees qualitatively with energy considerations of OFT (Butler and Bence 1984, Li et al. 1985, Wetterer 1989). In order to make such shifts, which deviate from ASM predictions, it has been suggested that the sunfish must be able to estimate absolute prey size (Li et al. 1985, O'Brien et al. 1985, Wetterer 1989). Our results indicate that this shift in favor of larger prey is true only for relatively large-sized sunfish and that prey selectivities by all sunfish size categories are in qualitative agreement with OFT.

Li et al. (1985) concluded that bluegill sunfish subjected to conditions of poor visual resolution feed according to apparent size. Given that small sunfish $(<27$ $\mathrm{mm}$ SL) have relatively poor visual capabilities as compared to larger sunfish, we therefore expected that small sunfish should feed according to apparent size. Contrary to expectations, small sunfish were more selective than was predicted by the apparent size model and showed a preference for small prey. Unlike the ASM, which adequately described diet selection only for fish of intermediate size (25-38 $\mathrm{mm}$ SL), the optimal foraging model (Mittelbach 1981, Werner et al. 1983) predicted correctly the change in selectivity from small to large prey as fish size increased.

Although the mean prey size attacked (and consumed) conformed more closely to energy maximization considerations of OFT than to relative encounter probabilities based on apparent prey size, sunfish diet breadths were greater than those predicted by OFT. All sunfish incorporated suboptimal prey in their diets. Stephens and Krebs (1986) emphasized that diet, as measured by stomach contents, and food preference in dichotomous choice situations are not strictly predicted by the optimal foraging "prey" model. The model assumes that net energy gain, handling time and encounter rate for each prey type are fixed for a particular prey assemblage and are not functions of the predetermined attack probabilities. While stomach contents provide a reasonable measure of attacks by large-sized, planktivorous sunfish because capture success is nearly $100 \%$, for less adept, small-sized sunfish, the distributions of attacks and prey in the diet differ. Our results show that the mean prey size attacked was correlated better with OFT predictions than was the mean prey size captured. Because OFT predicted that most sunfish size categories should specialize on only one or two prey size categories, diet breadths were not amenable to statistical examination by the chi-squared test.

Bence and Murdoch (1986) found that, relative to starved mosquitofish, prefed Gambusia increased their attacks on valuable prey, but did not exclude items outside the optimal set from their diets. They concluded that optimal diet models predict qualitatively that attacks on profitable prey should increase relative to attacks on less profitable prey as the abundance of profitable prey increases, but that the zero-one rule (predators should exclude from their diets those prey whose profitability is below some critical threshold) is not robust. This also was true for sunfish, but we did not examine how selectivity changes with the density of profitable prey or predator satiation.

Although violations of other assumptions of the OFT "prey" model (see Stephens and Krebs 1986) will certainly affect the accuracy of the model predictions, two factors may have particular relevance to our study: the assimilable fraction of prey energy content is taken to be a constant (Mittelbach 1981) and prey encounter is assumed to be sequential and a Poisson process (Stephens and Krebs 1986).

Because prey biomass is related directly to energy content, Werner and Hall (1974) stated that bluegill sunfish select prey to maximize the biomass ingested. For small sunfish, however, the relationship between ingested prey biomass and energy return may not be straightforward. If assimilation rates by small fish differ among prey size categories such that large prey are not digested efficiently, then, consuming small-sized prey is not suboptimal. Rather than maximizing biomass ingested per se, small bluegill may maximize net energy intake by selecting prey that can be digested efficiently. Mills et al. (1984) found that small perch, Perca flavescens, despite being capable of ingesting large prey, select intermediate-sized Daphnia. Because digestion is incomplete in young fish (Noble 1973, Mills and Forney 1981), Mills et al. suggested that young fish digest intermediate-sized prey more efficiently than large Daphnia. In addition to varying with prey size, digestion is likely to vary with prey type (Confer and O'Bryan 1989) and meal size. For example, the predatory fly larva, Chaoborus, digested copepods $49 \%$ faster than daphnids, and assimilation efficiency declined with increasing prey size and after ingesting large meals (Giguere 1981, 1986).

We examined the effects of differential prey digestion on the optimal diet sets by varying the assimilable fraction of energy content in the optimal foraging model. The assimilation efficiency for medium Daphnia was set at values from 10 to $70 \%$ in diets incorporating two (small and medium) prey size groups. When the assimilation efficiency of small prey was held constant at $70 \%$ and that of medium Daphnia $\left(A_{m}\right)$ was between 33 and $25 \%$, the optimal diet for $11-\mathrm{mm}$ sunfish switched from small and medium prey to only small prey. For 14-mm sunfish, the optimal diet set included only medium Daphnia when $A_{m}>33 \%$, included small and medium Daphnia for $33 \% \leq A_{m}<$ $25 \%$, and included only small Daphnia for $A_{m}=10 \%$. The optimal diet for $17-\mathrm{mm}$ fish shifted from only medium prey to small and medium prey when $A_{m} \leq$ $25 \%$ and to only small prey when $A_{m}=10 \%$. For most small sunfish size categories, the optimal diet set (given 
our parameterization of the model) is therefore remarkably constant for a wide range of assimilation efficiencies and changes only when the assimilable fraction of energy content for the larger prey size category is less than $\approx 33 \%$. In other words, the assimilation rates of the larger prey must be $\geq 2$ times lower than that of the smaller prey before the optimal diets change. However, further studies are necessary to determine sunfish assimilation rates of prey that differ in size and morphology, and their effects on optimal diet predictions.

A second and more significant deviation from the assumption of foraging models is that in nature, and in most laboratory studies, prey encounters are often simultaneous. When prey encounters are not sequential, partial preferences may occur (Stephens and Krebs 1986) and the observed diet breadths are broader than those predicted by the optimal foraging "prey" model. Still, abrupt thresholds often are not evident even when OFT model assumptions are met (Stephens and Krebs 1986).

The ASM provides a mechanism for making a choice between prey that are encountered simultaneously (Wetterer 1989) and qualitatively predicts broader diet breadths than those predicted by the optimal foraging "prey" model. Selecting prey by apparent size is likely to be more efficient than selecting prey at random (Butler and Bence 1984) and is thought to describe prey choice by several aquatic predators (see Wetterer 1989). In order to consider prey that are encountered simultaneously in a sequential manner, sunfish may use apparent prey size to direct their attention to an individual prey item and, subsequently, evaluate actual prey size and make a choice according to a minimum size threshold (Wetterer 1989). Yet, partial preferences remain problematic and are thought to result from perceptual constraints: prey that are outside the binocular field and are apparently larger than any prey within the binocular field (Wetterer 1989).

Partial preferences might be explained better by optimal foraging models that assume simultaneous prey encounters (Waddington and Holden 1979, Carlsson 1983, Stephens et al. 1986). If prey choices are mutually exclusive, the critical travel time (defined as the reciprocal of encounter rate) will determine whether a forager should prefer a prey of lower profitability. Quantitative laboratory testing of simultaneous encounter models for sunfish is certainly warranted, but may be a formidable task (Butler and Bence 1984). As these models require accurate estimates of the expected net energy gained from individual prey items, prey handling times and encounter rates, rigorous tests of the models necessitate that we understand fully how the parameters change with sunfish growth and meet the models' assumptions.

The visual system and factors that influence energy considerations, such as prey handling times and digestibility, constrain prey selection in small sunfish, but operate in contrasting manners. Our results show that small sunfish, despite their poor visual capabilities, are more selective in the laboratory than is predicted by the apparent size model. This increased selectivity for small prey is described better by OFT and at least in part reflects the costs due to increased prey handling times by small fish.

\section{ACKNOWLEDGMENTS}

This study was supported by USDA Hatch Project NY(C)183424 to N. G. Hairston. We thank P. Edds, D. Hambright, G. Mittelbach, C. Pierce, J. Presson, G. Stirling, P. Wimberger, and two reviewers for their input, and R. Dawley for his assistance with the electrophoretic analyses.

\section{Literature Cited}

Bence, J. R., and W. W. Murdoch. 1986. Prey size selection by the mosquitofish: relation to optimal diet theory. Ecology 67:324-336.

Blaxter, J. H. S. 1986. Development of sense organs and behavior of teleost larvae with special reference to feeding and predator avoidance. Transactions of the American Fisheries Society 115:98-114.

Blaxter, J. H. S., and M. Staines. 1970. Pure-cone retinae and retinomotor responses in larval teleosts. Journal of the Marine Biological Association of the United Kingdom 50: 449-460.

Breck, J. E., and M. J. Gitter. 1983. Effect of fish size on the reaction distance of bluegill (Lepomis macrochirus) sunfish. Canadian Journal of Fisheries and Aquatic Sciences 40:162-167.

Butler, S. M., and J. R. Bence. 1984. A diet model for planktivores that follow density-dependent rules for prey selection. Ecology 65:1885-1894.

Carlsson, A. 1983. Maximising energy delivery to dependent young: a field experiment with red-backed shrikes (Lanius collurio). Journal of Animal Ecology 52:697-704.

Confer, J. L., and L. M. O’Bryan. 1989. Changes in prey rank and preference by young planktivores for short-term and long-term ingestion periods. Canadian Journal of Fisheries and Aquatic Sciences 46:1026-1032.

Dawley, R. 1987. Hybridization and polyploidy in a community of three sunfish species. Copeia 1987: 326-335.

Eggers, D. M. 1982. Planktivore preference by prey size. Ecology 63:381-390.

Fernald, R. D. 1988. Aquatic adaptations in fish eyes. Pages 435-466 in J. Atema, R. R. Fay, A. N. Popper, and W. N. Tavolga, editors. Sensory biology of aquatic animals. Springer-Verlag, New York, New York, USA.

Giguere, L. A. 1981. Food assimilation efficiency as a function of temperature and meal size in larvae of Chaoborus trivittatus (Diptera: Chaoboridae). Journal of Animal Ecology 50:103-109.

1986. The estimation of crop evacuation rates in Chaoborus larvae (Diptera: Chaoboridae) using natural prey. Freshwater Biology 16:557-560.

Hairston, N. G., Jr., K. T. Li, and S. S. Easter, Jr. 1982. Fish vision and the detection of planktonic prey. Science 218: $1240-1242$.

Hofer, R., and A. Nasir Uddin. 1985. Digestive processes during the development of the roach, Rutilus rutilus $\mathrm{L}$. Journal of Fish Biology 26:683-689.

Hunter, J. R. 1981. Feeding ecology and predation of marine fish larvae. Pages 33-79 in R. Lasker, editor. Marine fish larvae. Washington Sea Grant, University of Washington Press, Seattle, Washington, USA.

Johns, P. R., and S. S. Easter, Jr. 1977. Growth of the adult goldfish eye. II. Increase in retinal cell number. Journal of Comparative Neurology 176:331-342. 
Lemly, A. D., and J. F. Dimmick. 1982. Growth of youngof-the-year centrarchids in relation to zooplankton in the littoral zone of lakes. Copeia 1982:305-321.

Li, J. L., and H. W. Li. 1979. Species-specific factors affecting predator-prey interactions of the copepod Acanthocyclops vernalis with its natural prey. Limnology and Oceanography 24:613-626.

Li, K. T., J. K. Wetterer, and N. G. Hairston, Jr. 1985. Fish size, visual resolution, and prey selectivity. Ecology 66: 1729-1735.

Mark, W., W. Wieser, and C. Hohenauer. 1989. Interactions between developmental processes, growth, and food selection in the larvae and juveniles of Rutilus rutilus (L.) (Cyprinidae). Oecologia (Berlin) 78:330-337.

Miller, T. J., L. B. Crowder, J. A. Rice, and E. A. Marschall. 1988. Larval size and recruitment mechanisms in fishes: toward a conceptual framework. Canadian Journal of Fisheries and Aquatic Sciences 45:1657-1670.

Mills, E. L., J. L. Confer, and R. C. Ready. 1984. Prey selection by young yellow perch: the influence of capture success, visual acuity, and prey choice. Transactions of the American Fisheries Society 113:579-587.

Mills, E. L., and J. L. Forney. 1981. Energetics, food consumption, and growth of young yellow perch in Oneida Lake, New York. Transactions of the American Fisheries Society 110:479-488.

Mittelbach, G. G. 1981. Foraging efficiency and body size: a study of optimal diet and habitat use by bluegills. Ecology 62:1370-1386.

- 1984. Predation and resource partitioning in two sunfishes (Centrarchidae). Ecology 65:499-513.

- 1986. Predator-mediated habitat use: some consequences for species interactions. Environmental Biology of Fishes 16:159-169.

Neave, D. A. 1984. The development of visual acuity in larval plaice (Pleuronectes platessa L.) and turbot (Scophthalamus maximus L.). Journal of Experimental Marine Biology and Ecology 78:167-175.

Noble, R. L. 1973. Evacuation rates of young yellow perch, Perca flavescens (Mitchill). Transactions of the American Fisheries Society 102:759-763.

O’Brien, W. J., B. Evans, and C. Leucke. 1985. Apparent size choice of zooplankton by bluegill sunfish: exceptions to the rule. Environmental Biology of Fishes 13:225-233.
O'Brien, W. J., N. A. Slade, and G. L. Vinyard. 1976. Apparent size as the determinant of prey selection by bluegill sunfish (Lepomis macrochirus). Ecology 57:1304-1310.

Osenberg, C. W., E. E. Werner, G. G. Mittelbach, and D. J. Hall. 1988. Growth patterns in bluegill (Lepomis macrochirus) and pumpkinseed (L. gibbosus) sunfish: environmental variation and the importance of ontogenetic niche shifts. Canadian Journal of Fisheries and Aquatic Sciences 45:17-26.

Schmidt, D., and W. J. O'Brien. 1982. Planktivorous feeding ecology of arctic grayling (Thymallus arcticus). Canadian Journal of Fisheries and Aquatic Sciences 39:475-482.

Schmitt, R. J., and S. J. Holbrook. 1984. Gape-limitation foraging tactics and prey size selectivity of two microcarnivorous species of fish. Oecologia (Berlin) 63:6-12.

Stephens, D. W., and J. R. Krebs. 1986. Foraging theory. Princeton University Press, Princeton, New Jersey, USA.

Stephens, D. W., J. F. Lynch, A. E. Sorensen, and C. Gordon. 1986. Preference and profitability: theory and experiment. American Naturalist 127:533-553.

Vinyard, G. L., and W. J. O'Brien. 1976. Effects of light and turbidity on the reactive distance of bluegill sunfish (Lepomis macrochirus). Journal of the Fisheries Research Board of Canada 33:2845-2849.

Waddington, K. D., and L. R. Holden. 1979. Optimal foraging: on flower selection by bees. American Naturalist 114: 179-186.

Werner, E. E. 1974. The fish size, prey size, handling time relation in several sunfishes and some implications. Journal of the Fisheries Research Board of Canada 31:1531-1536.

Werner, E. E., and D. J. Hall. 1974. Optimal foraging and the size selection of prey by the bluegill sunfish (Lepomis macrochirus). Ecology 55:1042-1052.

Werner, E. E., G. G. Mittelbach, D. J. Hall, and J. F. Gilliam. 1983. Experimental tests of optimal habitat use in fish: the role of relative habitat profitability. Ecology 64:1525-1539.

Wetterer, J. K. 1989. Mechanisms of prey choice by planktivorous fish: perceptual constraints and rules of thumb. Animal Behaviour 37:955-967.

Wetterer, J. K., and C. J. Bishop. 1985. Planktivore prey selection: the reactive field volume model vs. the apparent size model. Ecology 66:457-464. 